\title{
Successful Healing Treatment of Kneecap (Patellar) Dislocation of a Teen Female Patient Using Yoga Prana Vidya System Protocols without Surgery: A Case Report
}

\section{Meena Dholakia ${ }^{1}$, Ikshita Tandon ${ }^{2}$, Dhaval Dholakia ${ }^{3}$ and Venkata Satyanarayana Nanduri ${ }^{4 *}$}

${ }^{1}$ Certified YPV HEALER, Senior YPV Trainer, Ahmedabad, Gujarat, India

${ }^{2}$ Associate Certified YPV Healer, YPV L1 Trainer, New Delhi, India

${ }^{3}$ Yoga Prana Vidya Arhat Trainer and Practitioner, Ahmedabad, Gujarat, India

${ }^{4}$ Consultant, Research and Publications, Yoga Prana Vidya Ashram, Sri Ramana

Trust, Tamilnadu, India

*Corresponding Author: Venkata Satyanarayana Nanduri, Consultant, Research and Publications, Yoga Prana Vidya Ashram, Sri Ramana Trust, Tamilnadu, India.
Received: October 04, 2021

Published: October 16, 2021

(C) All rights are reserved by Venkata

Satyanarayana Nanduri., et al.

\begin{abstract}
Introduction: Kneecap (Patella) dislocation is a common injury experienced by sports persons and dancers, most common in females, and generally occurs in adolescents below 17 years of age. Usual diagnostic procedures include physical examination, followed by X-ray, CT scan and MRI. Patients experience severe pain, instability in the knee and difficulty to walk. Both conservative and surgical treatments are available and its choice depends on various factors. This paper presents a case of patellar dislocation conservatively treated using Yoga Prana Vidya System protocols without medication and combined with physiotherapy.

Materials and Methods: This is an in-depth case study of a female aged 19 years at first instance of patellar dislocation. A study of the medical reports and patient/parents' feed back is carried out and results assessed with a follow up after 6 months.

Results: Within 2-3 months of healing and supported by physiotherapy, fitness certificate was issued by the specialist orthopaedic surgeon after examining her knee condition. A follow up after 6 months revealed that the patient had not experienced any swelling or pain, was able to walk and climb steps normally, was able to sit cross legged on floor with ease, with no other symptoms or discomfort.

Conclusion: In this case study, it is observed that Yoga Prana Vidya healing helped the patient wholly in recovery stages 1, 2 and 3, enabling the patient lead normal life. Thus, YPV system has shown to be a good adjunct in the conservative treatment of first patellar dislocation.
\end{abstract}

Keywords: Kneecap (Patellar) Dislocation; Yoga Prana Vidya System ${ }^{\circledR}$; YPV ${ }^{\circledR}$; Energy Healing; Conservative Treatment

\section{Introduction}

Patellar dislocation

When the patella (knee cap) slips out of its normal position it is a knee injury, termed as patellar dislocation. The incidence of acute primary patellar dislocations is $2-3 \%$. Patellar dislocations are often associated with athletes, and is most common in females in the second decade of life. Re-dislocation rates after conservative management are estimated between 15 and 44\% [1]. A patellar dis-

Citation: Meena Dholakia., et al. "Successful Healing Treatment of Kneecap (Patellar) Dislocation of a Teen Female Patient Using Yoga Prana Vidya System Protocols without Surgery: A Case Report". Acta Scientific Women's Health 3.11 (2021): 15-20. 
location occurs by a lateral shift of the patella, leaving the trochlea groove of the femoral condyle. This mostly occurs as a disruption of the medial patellofemoral ligament [1].

Main complaints from patients include: Pain, Instability of the knee, and Locking of the knee after the trauma. Usual diagnostic procedures are, physical examination followed by X ray, CT scans and MRI scans [2]. Conservative treatment, as an approach to treating utilizing non-surgical treatment options, such as physical therapy, medication and injections, is the most common treatment after primary patellar dislocation. Primary acute patellofemoral dislocations should be managed conservatively with immobilization and rehabilitation, as the majority of these patients will do well without surgery. An MRI is necessary to assess for osteochondral lesions, as they are associated with a poor prognosis if not addressed. Surgical management would be considered in cases with recurrent dislocations, or when it is associated with patellofemoral symptoms.

This paper presents a case of patellar dislocation conservatively treated using Yoga Prana Vidya System protocols without medication and combined with physiotherapy.

\section{Yoga prana vidya (YPV) system}

Yoga Prana Vidya System is an integrated and holistic system of healing and treating a wide range of physical and mental health conditions. It integrates the science of breathing, healing, meditation and yoga etc., to provide a holistic wellness solution. It works around all four aspects of being - Physical, Emotional, Mental and Spiritual. YPV empowers an individual to self-heal and unleash their full potential by enabling them to overcome any blocks, tapping into their unexplored and un-actualized resources. The participants are taught techniques such as - Simple physical exercises, Rhythmic Yogic Breathing, forgiveness and peace meditation, values and principles of the practice of healing techniques. Thus, YPV is an integrated and holistic system of healing and evidence shows that it has been used successfully as complementary and alternative medicine to treat hundreds of cases of several physical and psychological illnesses including some difficult medical cases [310]. Yoga Prana Vidya system is no-touch no-drug healing process which can be delivered by a trained healer to not only proximally seated patient, but also one who is situated at a distance of hundreds or even thousands of Kilometres away. Some simple techniques of YPV have become very popular with patients because of the simplicity and ease with which pain reduction in any part of the body is achieved quickly and with sustained results for patients.

\section{Materials and Methods}

This is an in-depth case study method of investigation of the phenomenon, of a patellar dislocation case in which energy healing protocols of Yoga Prana Vidya were applied successfully. Relevant medical records of the patient are reviewed and patient feedback supported by the feedback from patient's parents are analysed.

\section{Case Report}

\section{Case background}

- Patient Name: XXX (name withheld for reasons of confidentiality), Age: 19, Gender: Female.

- Patient suffered knee cap dislocation in February 2019 (Annexure 1 and 2) and thereafter in February 2020. On both these occasions, the condition was managed with some medication and YPV healing.

- In October 2020, the patient again had severe pain of knee cap dislocation in the same knee (right leg). She was not able to walk at all (Annexure 3). They had to walk to visit the doctor and she managed to cross the street under repair by walking un-assisted because of Yoga Prana Vidya healing.

- They consulted the orthopaedic surgeon on $7^{\text {th }}$ October 2020 (Annexure 4), who prescribed some pain killers for the pain to subside. It was a very much painful process for her to walk to see the doctor. With all this pain, she was managing with YPV healing because pain killers were not giving her any relief. As per the doctor's advice her MRI was obtained on $8^{\text {th }}$ October 2020 (Annexure 5) and as per the orthopaedic surgeon's advice the patient was taken to a knee specialist Doctor (who does only knee surgeries, especially for sports injury).

- As per his diagnosis (Annexure 6-8) her ligaments supporting the knee cap were weak, hence should be fixed by operation immediately. He had suggested to go for surgery followed by 6 months of rest and then physiotherapy. The knee specialist indicated that the patient may not be able to walk properly in future if surgery was not conducted. He had also further informed that after she gets married and when she will conceive, she will not be able to take her own weight. He however added that after getting the knee surgery done, there are chances that the condition can relapse. 
- $\quad$ The patient and her parents discussed this matter with the senior most YPV healer on $16^{\text {th }}$ October 2020 and he advised to continue healing and observe the condition. The patient also opted to undergo further healing, instead of surgery.
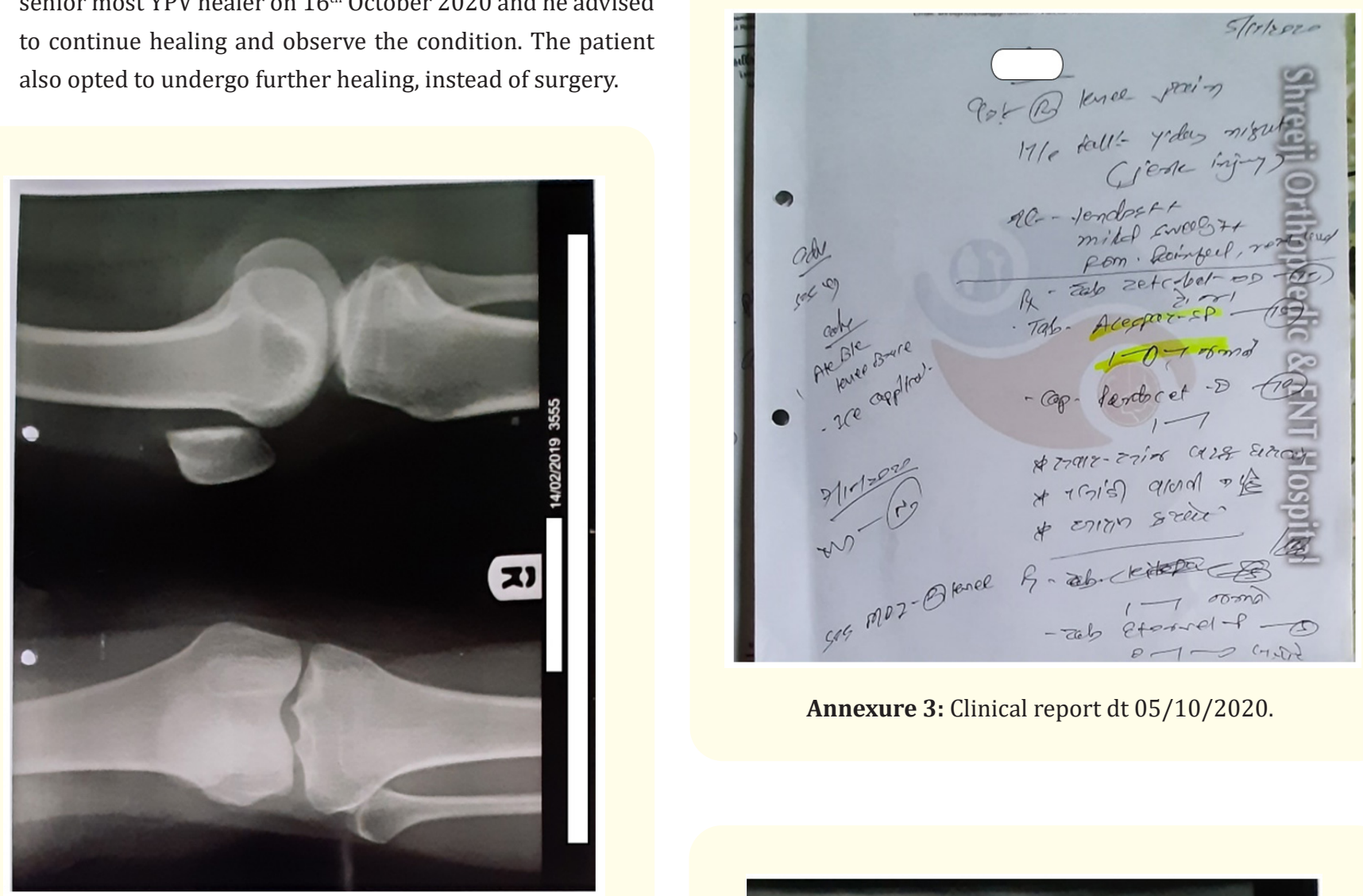

Annexure 3: Clinical report dt 05/10/2020.

Annexure 1: Right Knee X-ray dt 14/02/2019.
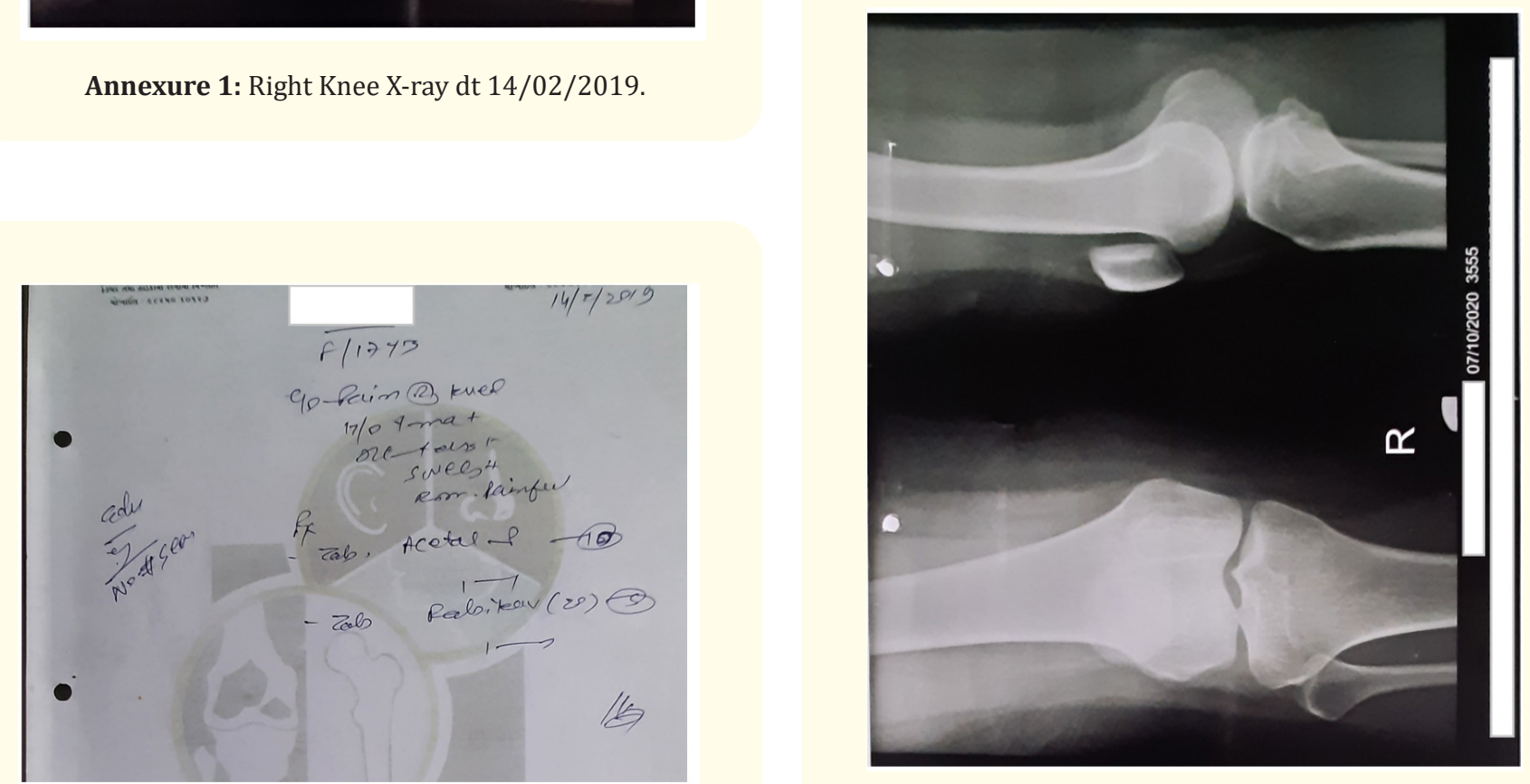

Annexure 2: Clinical report dated 14 /02/2019.

Annexure 4: X-Ray dt. 07/10/2020. 

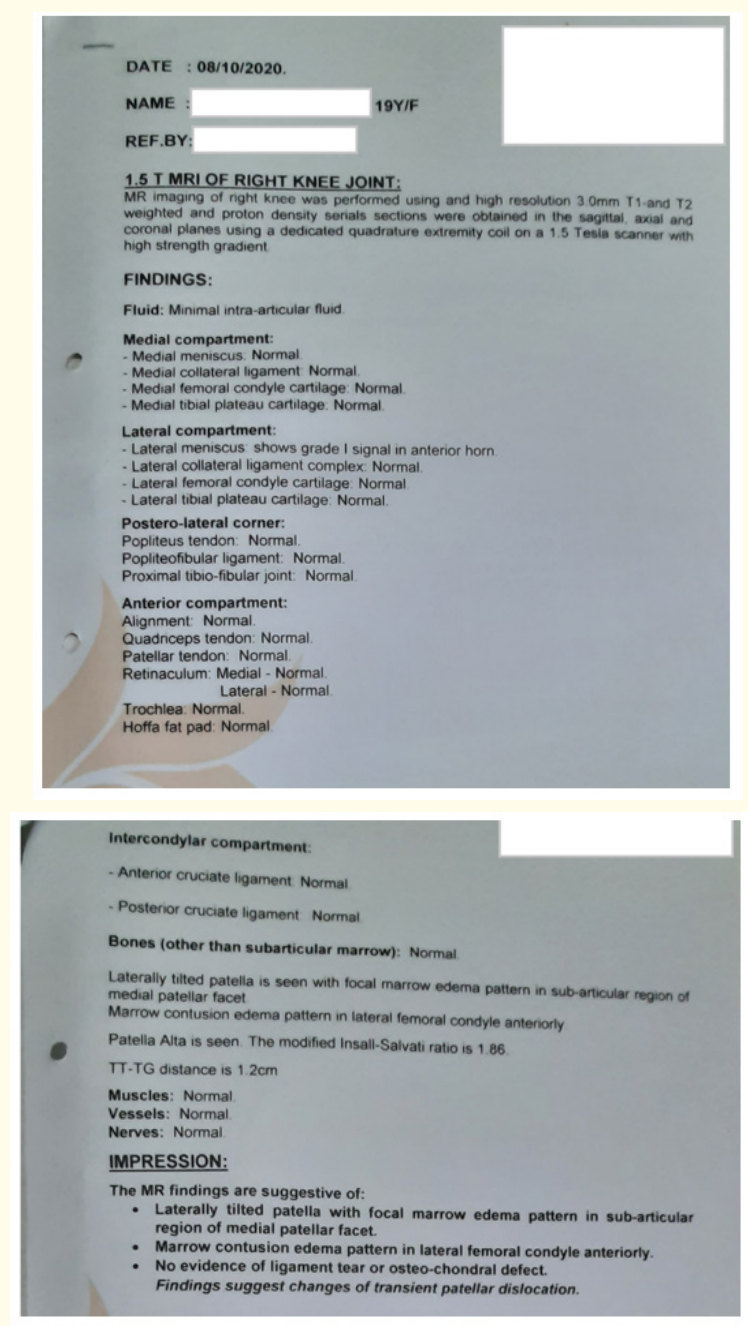

Annexure 5: MRI dt 08/10/2020.

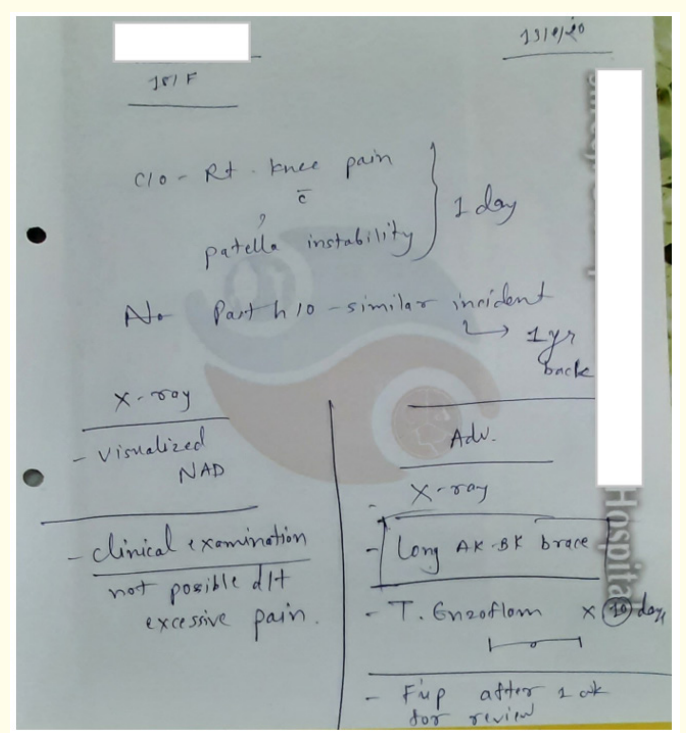

Annexure 6: Clinical report dt 13/02/2020.

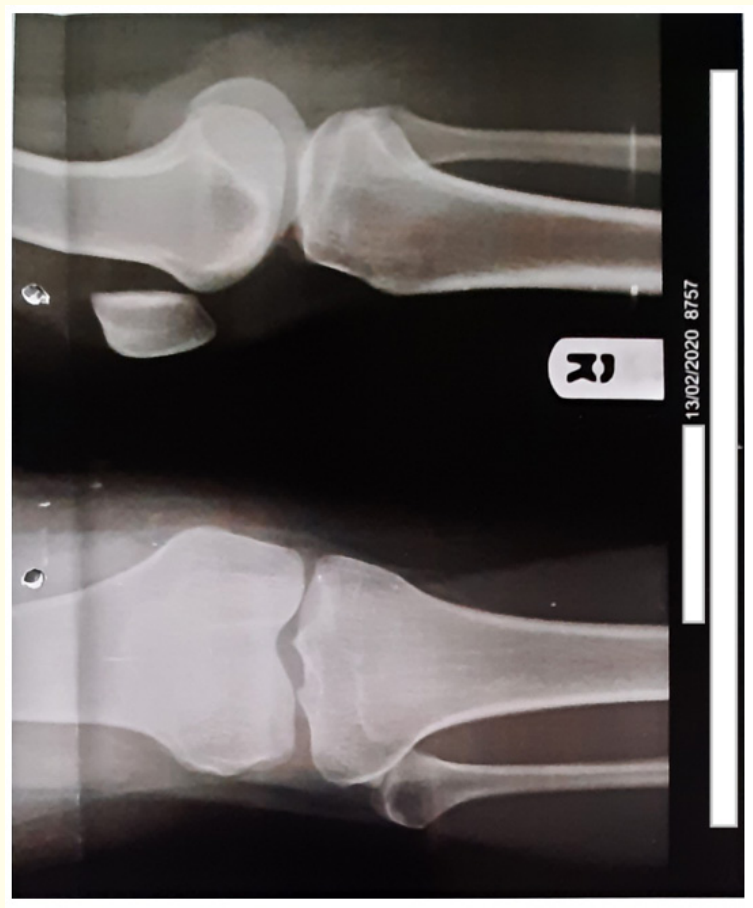

Annexure 7: X-Ray dt. 13/02/2020.

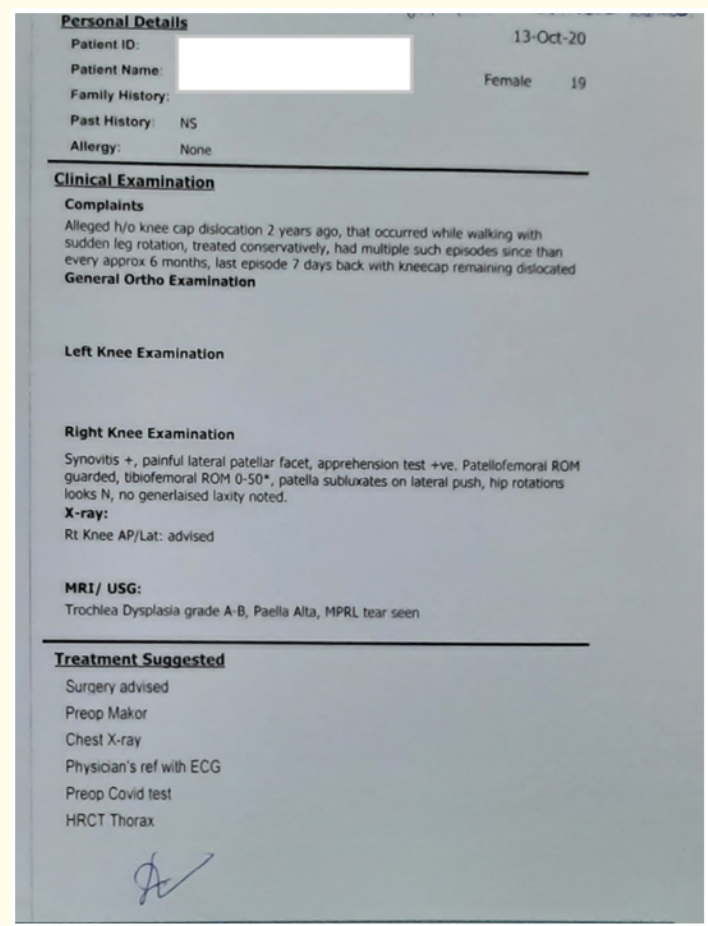

Annexure 8: Clinical report dt. 13/10/2020. 


\section{Full details of YPV protocols and healings}

A team of two healers were engaged to give healing treatment to the patient. The following protocols were used to treat the patient condition.

- YPV Psychotherapy

- Internal organ cleansing

- Blood cleansing.

Colour prana techniques were used to Localize the knee and the surrounding area; soak, Clean, and energize for reducing pain. Later on, when pain reduced substantially, energized for strengthening.

\section{Frequency of healing}

Initially the team healed 3 to 4 times a day (whole protocol once a day, rest of the time treated only affected part). After 1 month, healing was done once in a day.

\section{Results of YPV healings}

Since the patient decided not to undergo surgery, the healing team continued her healing throughout from 5th October 2020 to 15th February 2021 and within 2 to 3 months of healing physiotherapy also started. In April 2021, she was required to go to attend offline college, so her parents consulted the specialist (Annexure 9). After examining her knee condition, fitness certificate was issued by the specialist orthopaedic surgeon (Annexure 10). A follow up after 6 months revealed that she had not experienced any swelling or pain, able to walk and climb steps normally, was able to sit cross legged on floor with ease, and had no other symptoms or discomfort.

\section{Discussion}

The management of patella femoral instability is particularly complex, and despite the plentiful literature on this topic, robust scientific data are often lacking [11]. Conservative treatment and the subsequent rehabilitation program is generally based on specific formulated objectives, which can be divided into different stages:

- Stage 1: Resolution of pain, swelling and inflammation

- Stage 2: Recovery of joint motion and flexibility

- Stage 3: Recovery of muscle strength

- Stage 4: Recovery of motor patterns and coordination

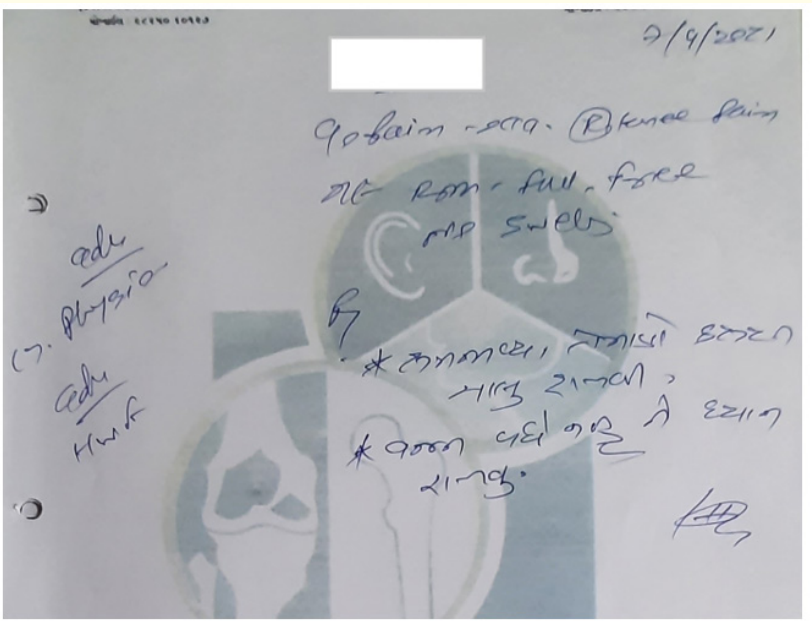

Annexure 9: Clinical report dt. 07/04/2021.

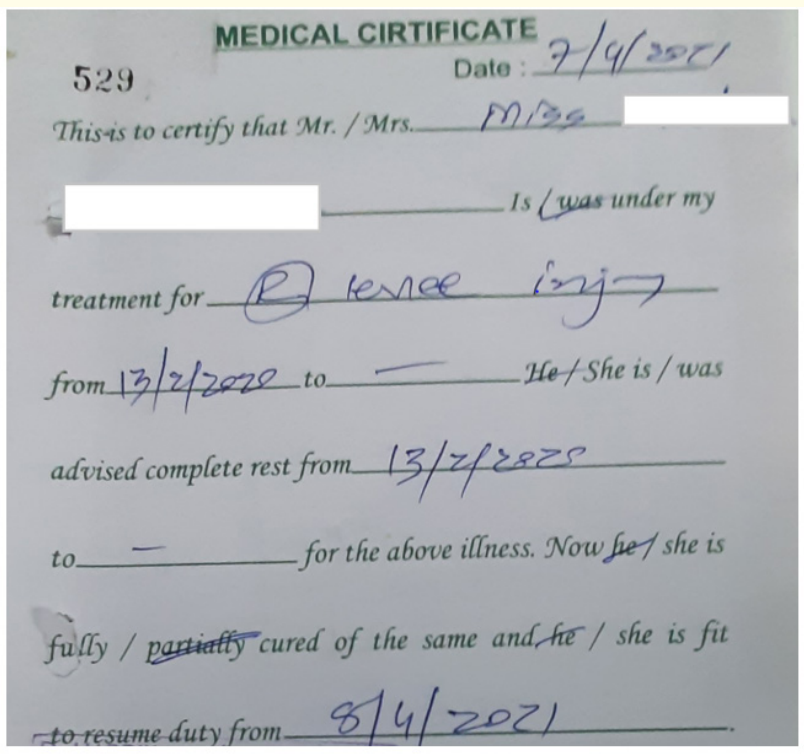

Annexure 10: Fitness certificate dt. 07/04/2021.

- Stage 5: Recovery of the sport-specific athletic action and return to sporting activity.

In this case study, it is observed that Yoga Prana Vidya healing helped the patient wholly in stages 1, 2 and 3, enabling the patient 
lead normal life. Thus, YPV system has shown to be a good adjunct in the conservative treatment of first dislocation.

According to Demirag., et al. [12]. Knee dislocations should be regarded as significant limb-threatening injuries. A comparative evaluation of surgical and conservative treatment by Demirag., et al. [12] showed that the mean range of motion of the knees (116 degrees versus 72 degrees; $\mathrm{p}<0.01$ ) and the mean Lysholm scores (84.6 versus $74 ; \mathrm{p}<0.01$ ) differed significantly between patients undergoing surgical and non-operative treatment. This was a longitudinal study of 46 months and the findings indicate that in cases of severity and complexity of this condition, surgical treatment afforded better results.

\section{Conclusion}

In this case study, it is observed that Yoga Prana Vidya healing helped the patient wholly in stages 1, 2 and 3, enabling the patient lead normal life. Thus, YPV system has shown to be a good adjunct in the conservative treatment of first patellar dislocation and future studies may be conducted on pilot scale samples or full-scale samples for a more generalised findings of efficacy of YPV in this segment of health condition.

\section{Acknowledgements}

Grateful acknowledgements to the healers, patient and her family for providing the case data and records, and to Sri Ramana Trust for using their copyrighted terms Yoga Prana Vidya System ${ }^{\circledR}$ and $\mathrm{YPV}^{\circledR}$.

\section{Conflicts of Interest}

None.

\section{Funding}

Nil.

\section{Bibliography}

1. Physiopedia. "Patellar dislocation" (2021).

2. Arora V and Kakkar A. "Recognition of Injury Patterns in Transient Lateral Patellar Dislocation on Magnetic Resonance Imaging". AMEI's Current Trends in Diagnosis and Treatment 5.1 (2021): 1-5.

3. J Neravetla and V S Nanduri. "A study into the successful treatment of some difficult medical cases using Yoga Prana Vidya (YPV) Healing System as alternative medicine". International Journal of Scientific and Engineering Research (2019): 1078827.
4. Ashalatha H Rajagopal., et al. "Diabetes Management and Control Using Yoga Prana Vidya (YPV) Healing System". Journal of Biology and Life Science 10.2 (2019).

5. V S Nanduri and Ms Chaitra N. "How the participants of a Yoga Prana Vidya (YPV) Eye Camp experienced vision improvements: A Case study". The Journal of Community Health Management 6.4 (2019).

6. N Jayachander Reddy and V S Nanduri. "A study of the effects of Yoga Prana Vidya one-month intensive residential programme for participants on their physical health, psychological well-being and improved immunity". International Journal of Research and Analytical Reviews (IJRAR) 7.2 (2020): 18-27.

7. N Jayachander Reddy and V S Nanduri. "Role of Yoga Prana Vidya (YPV) Healing Techniques in Emergency and First Aid: A Summary of Case Reports". International Journal of Medical Science and Health Research 4.3 133-146.

8. V S Nanduri. "Effectiveness of Yoga Prana Vidya practice protocols for health improvements and boosting immunity of seniors - A review". Journal of Bio Innovation 9.4 (2020): 583588.

9. V S Nanduri and Vishakha Karnani. Successful and speedy recovery of patients using Yoga Prana Vidya (YPV) Healing". Covid-19 1.4 (2020): 78-82.

10. V S Nanduri. "A Study on the Effects of Yoga Prana Vidya System (YPV) Intervention at workplace for Corporate Employees and Executives to alleviate Anxiety, Depression and Burnout; and participants' perceptions and experiences of the YPV Intervention". International Journal of Indian Psychology 8.3 (2020): 374-390.

11. Respizzi S and Cavallin R. "First patellar dislocation: from conservative treatment to return to sport". Joints 2.3 (2014): 141-145.

12. Demirağ B., et al. "Knee dislocations: an evaluation of surgical and conservative treatment". Ulusal Travma Ve Acil Cerrahi Dergisi 10.4 (2004): 239-244.

Volume 3 Issue 11 November 2021

(c) All rights are reserved by Venkata Satyanarayana Nanduri., et al. 